\title{
Investigation of Delamination in Thermal Barrier Coatings by Pulsed Thermography
}

\author{
by Kim W. ${ }^{*}$, Shrestha R. ${ }^{*}$ and Myoung S.** \\ * Department of Mechanical Engineering, Kongju National University, Cheonan, Republic of Korea \\ †Corresponding author: kwt@kongju.ac.kr \\ ${ }^{* *}$ R\&D Institute, Doosan Heavy Industries \& Construction, Changwon, Republic of Korea
}

\begin{abstract}
Thermal barrier coating (TBC) provides insulation to the base part and is widely used in aviation, power generation, and automotive industries to improve the service life of component being exposed to high temperatures. Delamination is a common failure mechanism of TBC and occurs after a critical number of thermal cycles. In this paper, detection of delamination in TBC system is presented. Pulsed thermography (PT) as a non-contact non-destructive evaluation (NDE) technique was employed to examine the delamination in TBCs. Time-dependent thermal images were used to characterize the delamination. The results showed that there was a relationship between the temperature distribution of the surface and delamination in TBCs.
\end{abstract}

\section{Introduction}

Thermal Barrier Coatings (TBCs) have been widely used in modern industries and applications to prolong the life of components. TBCs provide insulation to the base part and are widely used in aviation, power generation, and automotive industries to improve the service life of components being exposed to high temperatures. A typical TBC consists a nickelbased superalloy as a substrate material, intermetallic material as a bond coat, thermally grown oxide (TGO) and a ceramic top coat. Delamination is a common failure mechanism of TBC and occurs after a critical number of thermal cycles when the alumina layer has grown to a critical thickness. Therefore, there is a need for non-destructive testing (NDT) techniques to detect the delamination cracking. Among the NDT technologies, infrared thermography (IRT) deals with the acquisition and analysis of thermal information from non-contact, fast speed thermal imaging devices. IRT involves two classes, passive thermography, and active thermography. Based on the heating methods, active thermography could be further subdivided into pulsed thermography (PT), lock-in thermography (LIT), step heating, vibrothermography [1-4].

In this paper, test sample was excited by PT. The thermal variations of the material to this excitation is recorded which depends on different parameters of the material such as thermal conductivity, diffusivity, emissivity, and specific heat as well as the excitation used as the input. By analysing the thermal responses during heating and cooling process, the delamination present in TBC will be evaluated.

\section{Materials and methods}

In the present study, NiCoCrAIY bond coats and TBC systems with 8YSZ top coat was excited by PT. Fig. 1 and Fig. 2, respectively, shows a detail configuration of the test sample and experimental methodology considered in this study. The experimental PT system consisted of an Universal BALCAR (France), Power: $6400 \mathrm{~W}$-s as a heat source; BALCAR Light System, Nexus A 6400 (France) to synchronize the input and output signals; an IR camera (SC645, FLIR Systems, Sweden) with a $640 \times 480$ pixel resolution and a wavelength of $7.5-13 \mu \mathrm{m}$ to record the thermal response of the sample and a system controller (Computer).

\section{Results and Discussions}

The response to the applied heat flux was recorded over a period of $5 \mathrm{~s}$ using transient analysis. Fig. 3 shows the surface temperature decay curve of TBCs with different FCT cycles. As can be seen in Fig. 3, the temperature rise was faster than its decay because of very short impulse time. As the time elapsed, the temperature on the surface tends to reach equilibrium within $2 \mathrm{~s}$ for normal TBCs whereas for the TBC with delamination takes longer time. It was also observed that the surface temperature is relatively high when the TBCs FCT cycles increases.

\section{Conclusions}

In this work, study on investigation of delamination in TBC sample was done by using PT. The variation in the surface temperature distribution was sufficient for the qualitative evaluation of TBCs sample. The results showed that there was a relationship between the temperature distribution of the surface and delamination in TBCs. The method demonstrated potential in the investigation of delamination in TBCs samples. 


\section{Acknowledgement}

This work was supported by the National Research Foundation of Korea (NRF) grant funded by the Korean government (NRF-2016R1D1A1B03932587).

\section{REFERENCES}

[1] Ranjit S, Kim W. Thermal Analysis of Silicon Carbide Coating on a Nickel based Superalloy Substrate and Thickness Measurement of Top Layers by Lock-in Infrared Thermography. Journal of the Korean Society for Nondestructive Testing. - Vol. 37, no. 02, pp. 75-83, 2017.

[2] Ranjit S, Chung Y, Kim W. Thermal behavior variations in coating thickness using pulse phase thermography. Journal of the Korean Society for Nondestructive Testing. - Vol. 36, no. 04, pp. 259-265, 2016.

[3] Shrestha R, Kim W. Evaluation of coating thickness by thermal wave imaging: A comparative study of pulsed and lock-in infrared thermography-Part I: Simulation. Infrared Physics \& Technology. - Vol. 83, pp. 124-131, 2017.

[4] Shrestha R, Park J, Kim W. Application of thermal wave imaging and phase shifting method for defect detection in Stainless steel. Infrared Physics \& Technology. - Vol. 76, pp. 676-683, 2016.

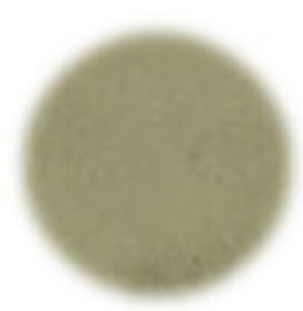

(a) 200 Cycle

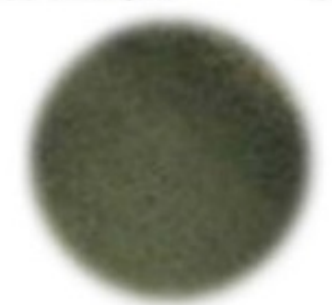

(e) 1000 Cycle

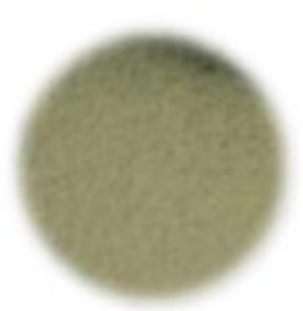

(b) 400 Cycle

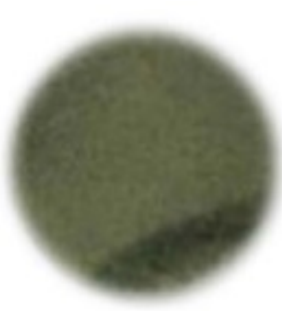

(c) 600 Cycle

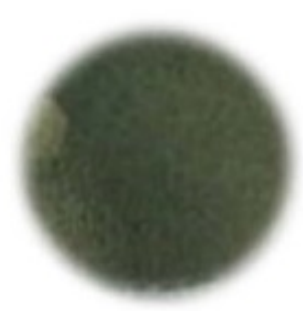

(d) 800 Cycle

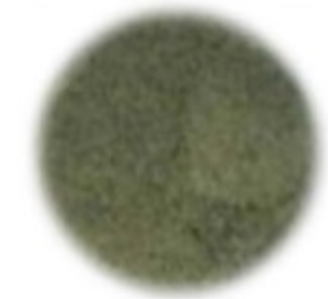

(f) 1200 Cycle

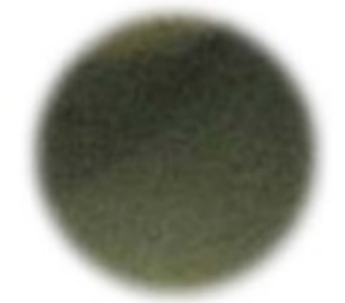

(g) 1200 Cycle with top coat Delamination

Fig. 1. TBCs after each thermal furnace cycling

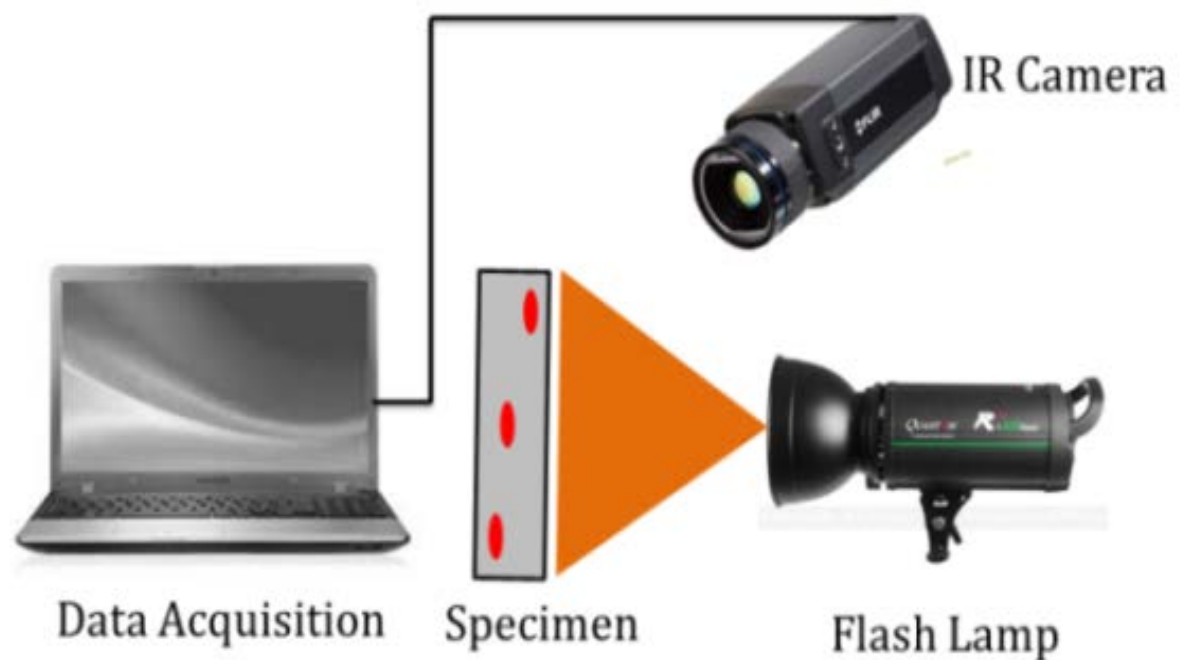

Fig. 2. Schematic of experimental setup for PT system 


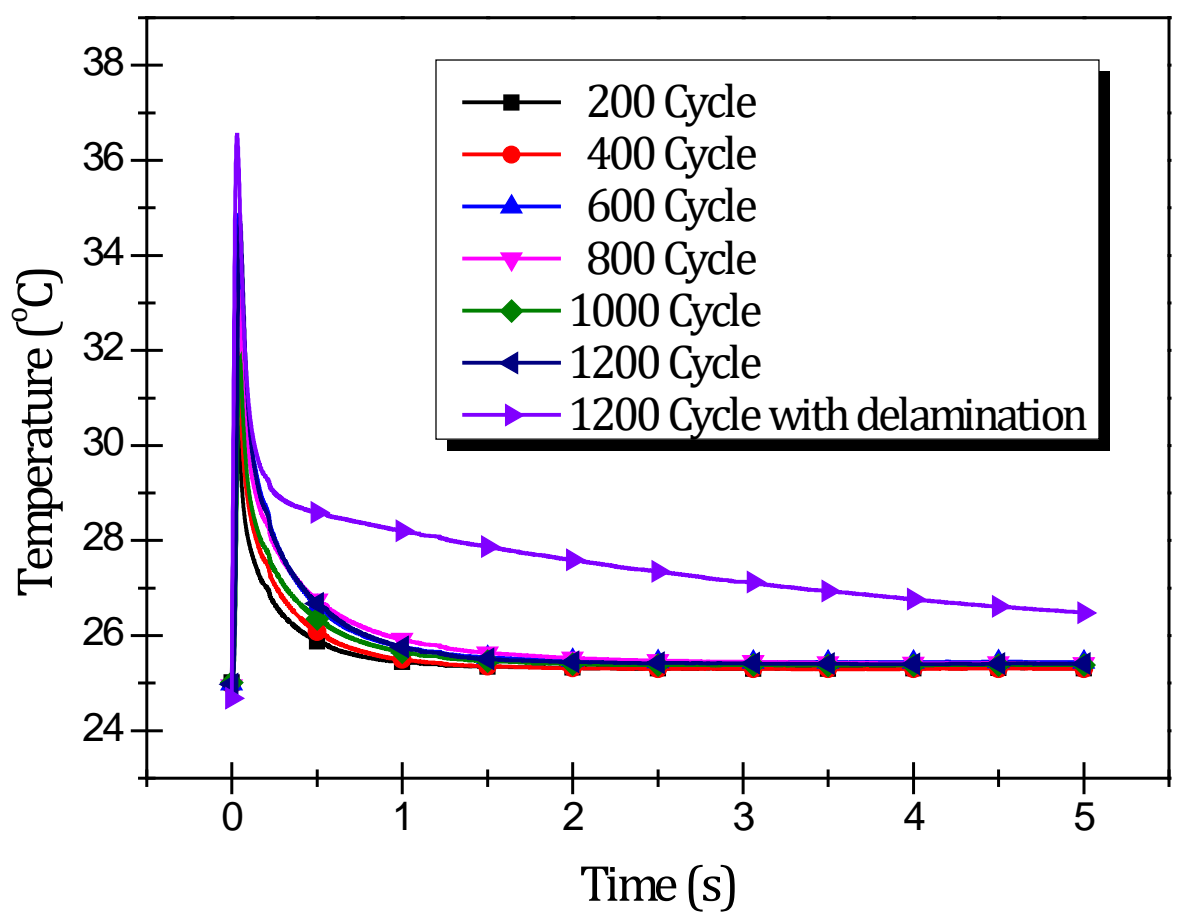

Fig. 3. Temperature evolution curve for the TBCs with different FCT cycles 